\title{
Vestibular stimulation does not diminish the desire for amputation
}

\author{
Lenggenhager, Bigna ; Hilti, L ; Palla, A ; Macauda, G ; Brugger, P
}

DOI: https://doi.org/10.1016/j.cortex.2014.02.004

Posted at the Zurich Open Repository and Archive, University of Zurich ZORA URL: https://doi.org/10.5167/uzh-100383

Journal Article

Accepted Version

Originally published at:

Lenggenhager, Bigna; Hilti, L; Palla, A; Macauda, G; Brugger, P (2014). Vestibular stimulation does not diminish the desire for amputation. Cortex, 54:210-212.

DOI: https://doi.org/10.1016/j.cortex.2014.02.004 


\section{Vestibular stimulation does not diminish the desire for amputation}

Bigna Lenggenhager ${ }^{1,3}$, Leonie Hilti ${ }^{1}$, Antonella Palla ${ }^{2}$, Gianluca Macauda ${ }^{1}$, Peter

$$
\text { Brugger }{ }^{1,3}
$$

\footnotetext{
${ }^{1}$ Neuropsychology Unit, Department of Neurology, University Hospital, Zurich, Switzerland

${ }^{2}$ Department of Neurology, University Hospital, Zurich, Switzerland

${ }^{3}$ Institute of Physiology and Zurich Center for Integrative Human Physiology (ZIHP),

University of Zurich
}

Correspondence to:

Bigna Lenggenhager

Neuropsychology

Department of Neurology

University Hospital Zurich

Frauenklinikstrasse 26

$\mathrm{CH}-8091$ Zurich, Switzerland

phone: +4144255 5584

email: bigna.lenggenhager@gmail.com

Word count: 992

Number of tables: 1

Key words: Body identity integrity disorder (BIID), amputation desire, xenomelia, caloric vestibular stimulation 


\section{INTRODUCTION}

While already more than a century ago, various disorders of the bodily self have been linked to the vestibular system (Bonnier, 1905), this link has only recently regained popularity. Modern studies generally suggest overlapping neural mechanisms in predominantly right parieto-insular areas. Corroboratively, it has been shown that artificial (caloric or galvanic) vestibular stimulation can temporarily normalize a variety of disorders of the bodily self (see e.g. Lopez, 2013 for detailed literature), including - most relevantly for the frame of this article - somatoparaphrenia (i.e. the denial of ownership for a body part (Rode et al., 1992)).

Against this background, Ramachandran and McGeoch (2007) suggested that caloric vestibular stimulation (CVS) might alleviate the desire for amputation in individuals suffering from xenomelia (also referred to as body identity integrity disorder (BIID) or apotemnophilia), i.e. the non-acceptance of one or several body parts in non-psychotic individuals. As psychological and pharmacological therapies have proven unsuccessful (e.g. Johnston \& Elliott, 2002), and the amputation of a healthy limb is associated with substantial ethical concerns (Muller, 2009), suggestions for alternative treatment are crucial and their empirical testing indispensable. We thus investigated in 13 persons with xenomelia, if left versus right cold CVS temporarily alleviates the intensity of the desire for amputation. As alterations in local body temperature might corroborate alterations in the feeling of limb ownership (Moseley et al., 2008), we further measured skin temperature. According to Ramachandran and McGeoch (2007), we hypothesized that left cold CVS, which activates predominantly the right hemisphere (Bottini et al., 1994) would decrease the momentary desire for amputation and increase body temperature for the affected body part.

\section{METHODS}

\section{Participants}

13 men with xenomelia (mean age $=48.3, \mathrm{SD}=13.1$ ) and 13 age-matched healthy men (mean age $=$ $49.2, S D=10.9$ ) took part in the experiment. Of the 13 subjects with xenomelia, 8 had an enduring amputation desire of the left leg, two of the right leg and three of both legs. The mean value on the subscale "pure amputation desire" of the Zurich Xenomelia Scale (Aoyama et al., 2012)) was 5.3 (SEM $0.2)$ out of 6 .

\section{Design and Procedure}

After an otological examination to ensure an intact tympanic membrane participants were oriented in supine position with the head inclined $30^{\circ}$ from the horizontal. Cold water $\left(22^{\circ}\right)$ irrigated the external auditory canal of one ear for the duration of approximately 4 minutes (see e.g. Mac Kay et al 2013). Right and left ears were counterbalanced. To verify successful vestibular stimulation, eye movements were recorded with video-oculography. Upon appearance of nystagmus, participants were asked to rate for all four limbs individually the momentary degree of estrangement on a scale from 0 (no estrangement) to 10 (extreme estrangement). These ratings were assessed once before 
stimulation and 4 times (every $40 \mathrm{sec}$ ) during CVS. During each rating skin temperature was measured using infrared thermometry (IRtek, IR15, Australia) in a counterbalanced order on eight body locations (for all four limbs above and below the elbow/knee, which corresponded to the level of desire for amputation).

\section{RESULTS}

\section{$7 \quad$ Rating of estrangement of a body part}

8 The momentary feeling of estrangement (Table 1) was not altered by the factor STIMULATION (left 9 cold CVS, right cold GVS, no stimulation) in either group.

Temperature

12 The results of the temperature measurements are reported in the SOM. Importantly, we did not find 13 any main or interaction effect of GROUP (healthy, wish for left leg amputation), suggesting that the 14 temperature as well as its modulation by caloric stimulation did not differ between healthy 15 participants and participants with a desire for left leg amputation.

\section{DISCUSSION AND CONCLUSION}

We set out to investigate if caloric vestibular stimulation can transiently normalize the perception of the bodily self in individuals suffering from xenomelia. Such normalization was repeatedly described in neurologically-caused disorders of the bodily self, notably also in disturbed feelings of ownership (somatoparaphrenia, (Rode et al., 1992)). As a consequence, and perhaps too hastily, the method was propagated as a cure of xenomelia (Muller, 2009).

The results in our group of 13 patients with a strong desire of amputation, however, suggest no significant impact of the procedure on body ownership as evidenced both by subjective report and objective changes in body temperature. In a novel field in which speculations abound, this negative finding is of utmost importance, the mere hypothesis that xenomelia could be influenced by CVS has already been taken as a springboard for musing about the potential effectiveness of brain stimulation (Muller, 2009). Documenting the lack of effectiveness of the procedure will not only restrain neuromythical thinking, but bears also consequences for developing a model of xenomelia and therapeutic approaches. We can only speculate why this hypothesis tested by a carefully designed experimental setup in a reasonably sized sample did not receive support. First of all, CVS is usually thought to normalize the perception of the bodily self (e.g. in somatoparaphrenia) through altering activity in core regions of the vestibular cortex such as the temporo-parietal and insular areas. Yet, recent fMRI data in individual with xenomelia suggest alterations in sensory mechanisms in premotor cortex rather than in posterior areas (van Dijk et al., 2013) - area that has also shown important illusory changes of ownership in healthy participants (e.g. Ehrsson et al., 2005). 
1 Secondly, the lack of an effect of CVS might also rely on the chronicity of the disorder. While there is

2 increasing evidence for a neurological basis, implication of social processes, which might continuously

3 strengthen the desire for amputation, has been proposed (Brugger et al., 2013). Moreover, and

4 contrary to patients with neurologically caused disorders of the bodily self, individuals suffering from

5 xenomelia often spend a great amount of time with simulating their desired body-state (Brugger et

6 al., 2013). Extensive simulation combined with the fact that the desire typically exists from early

7 childhood suggests that the altered body image might be too consolidated to be changed by short-

8 term vestibular stimulation. In conclusion, our data suggest that the previously drawn parallels

9 between somatoparaphrenia and xenomelia may have been overemphasized. Likewise, pure

10 neurological accounts of xenomelia, including the notion of a disturbed multisensory integration

11 might constitute oversimplifications of a condition that clearly deserves to be illuminated from an

12 interdisciplinary perspective.

13

14

\section{FUNDING}

16 BL and PB were funded by the Swiss National Science Foundation, grant \# 142601 and \# 17 320030_127480, respectively.

18 
Aoyama, A., Krummenacher, P., Palla, A., Hilti, L., \& Brugger, P. (2012). Impaired spatialtemporal integration of touch in xenomelia (body integrity identity disorder). Spat Cogn Comput, 12(2-3), 96-110.

Bonnier, P. (1905). L'Aschématie. Rev Neurol, 12, 605-609.

Bottini, G., Sterzi, R., Paulesu, E., Vallar, G., Cappa, S. F., Erminio, F., . . Frackowiak, R. S. (1994). Identification of the central vestibular projections in man: a positron emission tomography activation study. Exp Brain Res, 99(1), 164-169.

Brugger, P., Lenggenhager, B., \& Giummarra, M. J. (2013). Xenomelia: a social neuroscience view of altered bodily self-consciousness. Front Psychol, 4, 204.

Ehrsson, H. H., Holmes, N. P., \& Passingham, R. E. (2005). Touching a rubber hand: feeling of body ownership is associated with activity in multisensory brain areas. J Neurosci, 25(45), 10564-10573.

Johnston, J., \& Elliott, C. (2002). Healthy limb amputation: ethical and legal aspects. Clin Med, 2(5), 431-435.

Lopez, C. (2013). A neuroscientific account of how vestibular disorders impair bodily selfconsciousness. Front Integr Neurosci, 7(91).

McKay, R., Tamagni, C., Palla, A., Krummenacher, P., Hegemann, S.C., Straumann, D., Brugger, P. (2013) Vestibular stimulation attenuates unrealistic optimis. Cortex, 49(8),2272-5.

Moseley, G. L., Olthof, N., Venema, A., Don, S., Wijers, M., Gallace, A., \& Spence, C. (2008). Psychologically induced cooling of a specific body part caused by the illusory ownership of an artificial counterpart. Proc Natl Acad Sci U S A, 105(35), 1316913173.

Muller, S. (2009). Body Integrity Identity Disorder (BIID)-Is the Amputation of Healthy Limbs Ethically Justified? Am J Bioeth, 9(1), 36-43.

Ramachandran, V. S., \& McGeoch, P. (2007). Can vestibular caloric stimulation be used to treat apotemnophilia? Med Hypotheses, 69(2), 250-252.

Rode, G., Charles, N., Perenin, M. T., Vighetto, A., Trillet, M., \& Aimard, G. (1992). Partial remission of hemiplegia and somatoparaphrenia through vestibular stimulation in a case of unilateral neglect. Cortex, 28(2), 203-208.

van Dijk, M. T., van Wingen, G. A., van Lammeren, A., Blom, R. M., de Kwaasteniet, B. P., Scholte, H. S., \& Denys, D. (2013). Neural basis of limb ownership in individuals with body integrity identity disorder. PloS One, 8(8), e72212. 


\begin{tabular}{|c|c|c|c|c|c|c|c|c|}
\hline & \multicolumn{2}{|c|}{ Baseline } & \multicolumn{2}{l|}{ Left CVS } & \multicolumn{2}{l|}{ Right CVS } & \multicolumn{2}{l|}{ Friedmann (p-values) } \\
\hline $\begin{array}{c}\text { Healthy } \\
(\mathbf{n = 1 3 )}\end{array}$ & Left foot & Right foot & Left foot & Right foot & Left foot & Right foot & Left foot & Right foot \\
\hline $\begin{array}{c}\text { Amp Left } \\
(\mathbf{n}=\mathbf{8})\end{array}$ & $5.5(+/-0.8)$ & $0(+/-0)$ & $0(+/-0)$ & $0(+/-0)$ & $0(+/-0)$ & $0(+/-0)$ & $\mathrm{p}=1.00$ & $\mathrm{p}=1.00$ \\
\hline $\begin{array}{c}\text { Amp Right } \\
(\mathbf{n = 2})\end{array}$ & $0(+/-0)$ & $4.0(+/-0.7)$ & $0(+/-0)$ & $4.0(+/-0.7)$ & $0(+/-0)$ & $3.9(+/-0.7)$ & $\mathrm{p}=1.00$ & $\mathrm{p}=0.37$ \\
\hline $\begin{array}{c}\text { Amp Both } \\
(\mathbf{n = 3})\end{array}$ & $1.7(+/-1.2)$ & $1.8(+/-0.6)$ & $1.1(+/-1.4)$ & $1.5(+/-0.5)$ & $2.4(+/-1.5)$ & $2.1(+/-0.6)$ & $\mathrm{p}=0.10$ & $\mathrm{p}=0.71$ \\
\hline
\end{tabular}

3 Table 1: Mean scores (+/- SEM) for the question on "how much estrangement do you feel right now for the

4 foot " for both the left and the right foot during baseline, left CVS and right CVS. The last column includes the 5 Friedman test assessing the influence of condition (baseline, left CVS, right CVS) on the scores.

6

7

\begin{tabular}{|c|c|c|c|c|c|c|c|c|}
\hline & \multicolumn{2}{|c|}{ Baseline } & \multicolumn{2}{|l|}{ Left CVS } & \multicolumn{2}{|l|}{ Right CVS } & \multicolumn{2}{|c|}{ Friedmann (p-values) } \\
\hline & Left foot & Right foot & Left foot & Right foot & Left foot & Right foot & Left foot & Right foot \\
\hline Healthy & $31.0(+>-0.5)$ & $(+1-0)$ & $0(+1-0)$ & $0(+1-0)$ & $\stackrel{0(+1-0)}{=}$ & $\rho(+1-0)$ & $p=1.00$ & $\mathrm{p=1.00}$ \\
\hline$(n=13)$ & & & & & & & & \\
\hline Amp Left. & $30.3(+1-0.6)$ & $\rho(+1-0)$ & A.9. $9+(+-0.9)$ & $\rho(+\mid-0)$ & $4.8(+-0.9)$ & $0(+1-0)$ & $p=0.42$ & $\mathrm{p}=1.00$ \\
\hline$(n=8)$ & & & & & & & & \\
\hline Amp Right & $\stackrel{0(+1-0)}{a}$ & 4. $0(+1-0.7)$ & $\stackrel{0(+1-0)}{a}$ & $4.0(+-0.7)$ & مl(+1-0) & $3.9+(+-0.7)$ & $p=1.00$ & $\mathrm{p}=0.37$ \\
\hline$(n=2)$ & & & & & & & & \\
\hline Amp Both & & $1.8(+\mid-0.6)$ & & & & & & \\
\hline & $1.7(+\mid-1.2)$ & & $1.1(+/-1.4)$ & $1.5(+-0.5)$ & $2.4(+-1.5)$ & $2.1+(+-0.6)$ & $\mathrm{p}=0.10$ & $\mathrm{p}=0.71$ \\
\hline$(n=3)$ & & & & & & & & \\
\hline
\end{tabular}

8

Formatiert

[1]

Formatiert: Schriftart: 7 Pt., Nicht Fett, Nicht Kursiv

Formatiert

.. [2]

Formatiert: Schriftart: $7 \mathrm{Pt}$

Formatiert: Schriftart: 7 Pt., Nicht Fett, Nicht Kursiv

Formatiert

Formatiert

Formatiert

Formatiert

Formatiert

... [7]

Formatiert: Schriftart: 7 Pt., Nicht

Fett, Nicht Kursiv

Formatiert

Formatiert

Formatiert

Formatiert

Formatiert

Formatiert

Formatiert

Formatiert

Formatiert

Formatiert

Formatiert

Formatier

Formatiert

Formatiert

Formatiert

Formatiert

Formatiert

Formatiert

Formatiert

Formatiert: Schriftart: $7 \mathrm{Pt}$.

Formatiert

Formatiert: Schriftart: $7 \mathrm{Pt}$

Formatiert: Schriftart: 7 Pt., Nicht

Fett, Nicht Kursiv

Formatiert

Formatiert

Formatiert

Formatiert

Formatiert

Formatiert: Schriftart: 7 Pt., Nicht Kursiv 


\begin{tabular}{|c|c|c|}
\hline Seite 6: [1] Formatiert & Bigna Lenggenhager & 15.01.2014 16:11:00 \\
\hline \multicolumn{3}{|l|}{ Schriftart: 7 Pt. } \\
\hline Seite 6: [1] Formatiert & Bigna Lenggenhager & 15.01.2014 16:11:00 \\
\hline \multicolumn{3}{|l|}{ Schriftart: 7 Pt. } \\
\hline Seite 6: [2] Formatiert & Bigna Lenggenhager & 15.01.2014 16:11:00 \\
\hline \multicolumn{3}{|l|}{ Schriftart: 7 Pt. } \\
\hline Seite 6: [2] Formatiert & Bigna Lenggenhager & 15.01.2014 16:11:00 \\
\hline \multicolumn{3}{|l|}{ Schriftart: 7 Pt. } \\
\hline Seite 6: [3] Formatiert & Bigna Lenggenhager & 15.01.2014 16:11:00 \\
\hline \multicolumn{3}{|l|}{ Schriftart: 7 Pt. } \\
\hline Seite 6: [3] Formatiert & Bigna Lenggenhager & 15.01.2014 16:11:00 \\
\hline \multicolumn{3}{|l|}{ Schriftart: 7 Pt. } \\
\hline Seite 6: [4] Formatiert & Bigna Lenggenhager & 15.01.2014 16:11:00 \\
\hline \multicolumn{3}{|l|}{ Schriftart: 7 Pt. } \\
\hline Seite 6: [4] Formatiert & Bigna Lenggenhager & 15.01.2014 16:11:00 \\
\hline \multicolumn{3}{|l|}{ Schriftart: 7 Pt. } \\
\hline Seite 6: [5] Formatiert & Bigna Lenggenhager & 15.01.2014 16:11:00 \\
\hline \multicolumn{3}{|l|}{ Schriftart: 7 Pt. } \\
\hline Seite 6: [5] Formatiert & Bigna Lenggenhager & 15.01.2014 16:11:00 \\
\hline \multicolumn{3}{|l|}{ Schriftart: 7 Pt. } \\
\hline Seite 6: [6] Formatiert & Bigna Lenggenhager & 15.01.2014 16:11:00 \\
\hline \multicolumn{3}{|l|}{ Schriftart: 7 Pt. } \\
\hline Seite 6: [6] Formatiert & Bigna Lenggenhager & 15.01.2014 16:11:00 \\
\hline \multicolumn{3}{|l|}{ Schriftart: 7 Pt. } \\
\hline Seite 6: [7] Formatiert & Bigna Lenggenhager & 15.01.2014 16:11:00 \\
\hline \multicolumn{3}{|l|}{ Schriftart: 7 Pt. } \\
\hline Seite 6: [7] Formatiert & Bigna Lenggenhager & 15.01.2014 16:11:00 \\
\hline \multicolumn{3}{|l|}{ Schriftart: 7 Pt. } \\
\hline Seite 6: [8] Formatiert & Bigna Lenggenhager & 15.01.2014 16:11:00 \\
\hline \multicolumn{3}{|l|}{ Schriftart: 7 Pt. } \\
\hline Seite 6: [8] Formatiert & Bigna Lenggenhager & 15.01.2014 16:11:00 \\
\hline \multicolumn{3}{|l|}{ Schriftart: 7 Pt. } \\
\hline Seite 6: [9] Formatiert & Bigna Lenggenhager & 15.01.2014 16:11:00 \\
\hline \multicolumn{3}{|l|}{ Schriftart: 7 Pt. } \\
\hline Seite 6: [9] Formatiert & Bigna Lenggenhager & 15.01.2014 16:11:00 \\
\hline \multicolumn{3}{|l|}{ Schriftart: 7 Pt. } \\
\hline Seite 6: [10] Formatiert & Bigna Lenggenhager & 15.01.2014 16:11:00 \\
\hline \multicolumn{3}{|l|}{ Schriftart: 7 Pt. } \\
\hline Seite 6: [10] Formatiert & Bigna Lenggenhager & 15.01.2014 16:11:00 \\
\hline \multicolumn{3}{|l|}{ Schriftart: 7 Pt. } \\
\hline Seite 6: [11] Formatiert & Bigna Lenggenhager & 15.01.2014 16:11:00 \\
\hline
\end{tabular}

Schriftart: 7 Pt. 
Schriftart: $7 \mathrm{Pt}$.

Seite 6: [12] Formatiert

Bigna Lenggenhager

15.01.2014 16:11:00

Schriftart: 7 Pt.

Seite 6: [12] Formatiert

Bigna Lenggenhager

15.01.2014 16:11:00

Schriftart: 7 Pt.

Seite 6: [13] Formatiert

Bigna Lenggenhager

15.01.2014 16:11:00

Schriftart: 7 Pt.

Seite 6: [13] Formatiert

Bigna Lenggenhager

15.01.2014 16:11:00

Schriftart: 7 Pt.

Seite 6: [14] Formatiert

Bigna Lenggenhager

15.01.2014 16:11:00

Schriftart: 7 Pt.

Seite 6: [14] Formatiert

Bigna Lenggenhager

15.01.2014 16:11:00

Schriftart: $7 \mathrm{Pt}$.

Seite 6: [15] Formatiert

Bigna Lenggenhager

15.01.2014 16:11:00

Schriftart: 7 Pt.

Seite 6: [15] Formatiert

Bigna Lenggenhager

15.01.2014 16:11:00

Schriftart: 7 Pt.

Seite 6: [16] Formatiert

Bigna Lenggenhager

15.01.2014 16:11:00

Schriftart: 7 Pt.

Seite 6: [16] Formatiert

Bigna Lenggenhager

15.01.2014 16:11:00

Schriftart: 7 Pt.

Seite 6: [17] Formatiert

Bigna Lenggenhager

15.01.2014 16:11:00

Schriftart: 7 Pt.

Seite 6: [17] Formatiert

Bigna Lenggenhager

15.01.2014 16:11:00

Schriftart: 7 Pt.

Seite 6: [18] Formatiert

Bigna Lenggenhager

15.01.2014 16:11:00

Schriftart: 7 Pt.

Seite 6: [18] Formatiert

Bigna Lenggenhager

15.01.2014 16:11:00

Schriftart: 7 Pt.

Seite 6: [19] Formatiert

Bigna Lenggenhager

15.01.2014 16:11:00

Schriftart: $7 \mathrm{Pt}$.

Seite 6: [19] Formatiert

Bigna Lenggenhager

15.01.2014 16:11:00

Schriftart: 7 Pt.

Seite 6: [20] Formatiert

Bigna Lenggenhager

15.01.2014 16:11:00

Schriftart: 7 Pt.

Seite 6: [20] Formatiert

Bigna Lenggenhager

15.01.2014 16:11:00

Schriftart: $7 \mathrm{Pt}$.

Seite 6: [21] Formatiert

Bigna Lenggenhager

15.01.2014 16:11:00

Schriftart: 7 Pt.

Seite 6: [21] Formatiert

Bigna Lenggenhager

15.01.2014 16:11:00 
Schriftart: 7 Pt.

Seite 6: [22] Formatiert

Bigna Lenggenhager

15.01.2014 16:11:00

Schriftart: 7 Pt.

Seite 6: [22] Formatiert

Bigna Lenggenhager

15.01.2014 16:11:00

Schriftart: 7 Pt.

Seite 6: [23] Formatiert

Bigna Lenggenhager

15.01.2014 16:11:00

Schriftart: $7 \mathrm{Pt}$.

Seite 6: [23] Formatiert

Bigna Lenggenhager

15.01.2014 16:11:00

Schriftart: $7 \mathrm{Pt}$.

Seite 6: [24] Formatiert

Bigna Lenggenhager

15.01.2014 16:11:00

Schriftart: 7 Pt.

Seite 6: [24] Formatiert

Bigna Lenggenhager

15.01.2014 16:11:00

Schriftart: 7 Pt.

Seite 6: [25] Formatiert

Bigna Lenggenhager

15.01.2014 16:11:00

Schriftart: $7 \mathrm{Pt}$.

Seite 6: [25] Formatiert

Bigna Lenggenhager

15.01.2014 16:11:00

Schriftart: 7 Pt.

Seite 6: [26] Formatiert

Bigna Lenggenhager

15.01.2014 16:11:00

Schriftart: 7 Pt.

Seite 6: [26] Formatiert

Bigna Lenggenhager

15.01.2014 16:11:00

Schriftart: $7 \mathrm{Pt}$.

Seite 6: [27] Formatiert

Bigna Lenggenhager

15.01.2014 16:11:00

Schriftart: $7 \mathrm{Pt}$.

Seite 6: [27] Formatiert

Bigna Lenggenhager

15.01.2014 16:11:00

Schriftart: 7 Pt.

Seite 6: [28] Formatiert

Bigna Lenggenhager

15.01.2014 16:11:00

Schriftart: $7 \mathrm{Pt}$.

Seite 6: [28] Formatiert

Bigna Lenggenhager

15.01.2014 16:11:00

Schriftart: 7 Pt.

Seite 6: [29] Formatiert

Bigna Lenggenhager

15.01.2014 16:11:00

Schriftart: 7 Pt.

Seite 6: [29] Formatiert

Bigna Lenggenhager

15.01.2014 16:11:00

Schriftart: 7 Pt.

Seite 6: [30] Formatiert

Bigna Lenggenhager

15.01.2014 16:11:00

Schriftart: 7 Pt.

Seite 6: [30] Formatiert

Bigna Lenggenhager

15.01.2014 16:11:00

Schriftart: 7 Pt.

Seite 6: [31] Formatiert

Bigna Lenggenhager

15.01.2014 16:11:00

Schriftart: 7 Pt.

Seite 6: [31] Formatiert

Bigna Lenggenhager

15.01.2014 16:11:00

Schriftart: $7 \mathrm{Pt}$. 
